\title{
Important Aspects of Geometric Numerical Integration
}

\author{
Ernst Hairer $^{1}$
}

\author{
Received September 30, 2003; accepted (in revised form) February 10, 2004
}

\begin{abstract}
At the example of Hamiltonian differential equations, geometric properties of the flow are discussed that are only preserved by special numerical integrators (such as symplectic and/or symmetric methods). In the 'non-stiff' situation the long-time behaviour of these methods is well-understood and can be explained with the help of a backward error analysis. In the highly oscillatory ('stiff') case this theory breaks down. Using a modulated Fourier expansion, much insight can be gained for methods applied to problems where the high oscillations stem from a linear part of the vector field and where only one (or a few) high frequencies are present. This paper terminates with numerical experiments at space discretizations of the sine-Gordon equation, where a whole spectrum of frequencies is present.
\end{abstract}

KEY WORDS: Geometric numerical integration; Hamiltonian systems; reversible differential equations; backward error analysis; energy conservation; modulated Fourier expansion; adiabatic invariants; sine-Gordon equation.

\section{INTRODUCTION}

After the pioneering contributions around the turn from the 19th to the 20 th century, and after the successful period in the 70 s and 80 s on stiff problems, the numerical solution of ordinary differential equations is again an active field of research. One of the main interests is now the subject of geometric numerical integration, which is synonymous with structurepreserving integration of differential equations.

This survey concentrates on the structure-preserving integration of Hamiltonian systems. A detailed presentation of further results on this topic can be found in the recent monograph [9].

\footnotetext{
${ }^{1}$ Section de Mathématiques, 2-4, rue du Lièvre, CH-1211 Genève 24, Switzerland. E-mail: Ernst.Hairer@math.unige.ch
} 


\subsection{Geometric Structures in Hamiltonian Systems}

Hamiltonian systems are differential equations of the form:

$$
\dot{p}=-\nabla_{q} H(p, q), \quad \dot{q}=\nabla_{p} H(p, q),
$$

where $H: \mathbb{R}^{d} \times \mathbb{R}^{d} \rightarrow \mathbb{R}$, and the dimension $d$ is the number of degrees of freedom. In applications the Hamiltonian is often given in the form:

$$
H(p, q)=\frac{1}{2} p^{T} M(q)^{-1} p+U(q)
$$

with a positive definite symmetric mass matrix $M(q)$ and a potential $U(q)$. In this situation, the function $H(p, q)$ represents the total energy of the system. Such problems arise in mechanics, astrophysics, molecular dynamics, and many other sciences.

Due to their special structure, Hamiltonian systems have several interesting properties (in the following we denote the flow of the system, mapping an initial value $y=(p, q)$ onto the solution at time $t$, by $\left.\varphi_{t}(y)\right)$ :

(P1) the group property $\varphi_{t} \circ \varphi_{s}=\varphi_{t+s}$ is satisfied by every differential equation; in particular, one has

$$
\varphi_{t} \circ \varphi_{-t}=\varphi_{0}=\text { identity, }
$$

(P2) the Hamiltonian $H(p, q)$ is constant along solutions of (1) which means that the total energy is a conserved quantity,

(P3) the flow $\varphi_{t}$ of (1) is a symplectic transformation, i.e.

$$
\varphi_{t}^{\prime}(y)^{T} J \varphi_{t}^{\prime}(y)=J \quad \text { for } \quad t \geqslant 0, \quad J=\left(\begin{array}{rr}
0 & I \\
-I & 0
\end{array}\right),
$$

where the prime in $\varphi_{t}^{\prime}(y)$ denotes the derivation with respect to $y$. Due to $\operatorname{det} \varphi_{t}^{\prime}(y)=1$, this implies that the flow is volumepreserving,

$$
\mu\left(\varphi_{t}(A)\right)=\mu(A) \text { for } t \geqslant 0
$$

and for systems with one degree of freedom symplecticity turns out to be equivalent with area-preservation of the flow $\varphi_{t}$,

(P4) if $H(-p, q)=H(p, q)$, the flow $\varphi_{t}$ is $\rho$-reversible with respect to the reflection $\rho(p, q)=(-p, q)$, i.e. it satisfies

$$
\left(\rho \circ \varphi_{t}\right)(y)=\left(\varphi_{t}^{-1} \circ \rho\right)(y) \text { for all } t \text { and all } y \text {. }
$$

It is natural to look for numerical methods that satisfy one or several of these properties. 


\subsection{Geometric Integrators}

A numerical method for solving ordinary differential equations is a mapping $\Phi_{h}$ defined on the phase space that approximates the time- $h$ flow $\varphi_{h}$; it is of order $r$ if $\Phi_{h}(y)=\varphi_{h}(y)+\mathcal{O}\left(h^{r+1}\right)$. The numerical approximation at time $t=n h$ is obtained by $y_{n}=\Phi_{h}\left(y_{n-1}\right)$. Motivated by the previous section, the following properties are of interest:

(S1) the method is symmetric if it satisfies

$$
\Phi_{h} \circ \Phi_{-h}=\text { identity, }
$$

(S2) it is energy-preserving if along numerical solutions of (1)

$$
H\left(p_{n}, q_{n}\right)=\text { const },
$$

(S3) it is called symplectic if $\Phi_{h}$ satisfies

$$
\Phi_{h}^{\prime}(y)^{T} J \Phi_{h}^{\prime}(y)=J,
$$

(S4) it is $\rho$-reversible if, for $H(-p, q)=H(p, q)$,

$$
\left(\rho \circ \Phi_{h}\right)(y)=\left(\Phi_{h}^{-1} \circ \rho\right)(y) \text { for all } h \text { and all } y .
$$

Note that by the property

$$
\left(\rho \circ \Phi_{h}\right)(y)=\left(\Phi_{-h} \circ \rho\right)(y),
$$

which is satisfied by all standard methods, $\rho$-reversibility (S4) is equivalent to symmetry (S1). A numerical method that satisfies one or several of these properties is called a geometric integrator.

The most important geometric integrator is the so-called StörmerVerlet method (cf. [10]). It is the composition of a half-step of the partitioned Euler method (explicit in $q$, implicit in $p$ ) with a half-step of its adjoint (explicit in $p$, implicit in $q$ ) and thus given by the formulae

$$
\begin{aligned}
p_{n+1 / 2} & =p_{n}-\frac{h}{2} \nabla_{q} H\left(p_{n+1 / 2}, q_{n}\right), \\
q_{n+1} & =q_{n}+\frac{h}{2}\left(\nabla_{p} H\left(p_{n+1 / 2}, q_{n}\right)+\nabla_{p} H\left(p_{n+1 / 2}, q_{n+1}\right)\right), \\
p_{n+1} & =p_{n+1 / 2}-\frac{h}{2} \nabla_{q} H\left(p_{n+1 / 2}, q_{n+1}\right) .
\end{aligned}
$$

Direct verification shows that this method is symmetric (S1) and symplectic (S3). Since it satisfies (11), it is also $\rho$-reversible (S4). It does not satisfy (S2), even not for the harmonic oscillator $H(p, q)=\frac{1}{2}\left(p^{2}+q^{2}\right)$, but it 
approximately conserves the Hamiltonian over extremely long time intervals as we shall see in Sec. 2.

The only disadvantage of the Störmer-Verlet method (12) is its low order 2, and it is therefore inefficient for high-accuracy computations (as needed, for example, in planetary motion simulation). Much research of the last decade has been devoted to the construction and discussion of higher order geometric integrators (such a composition methods, implicit Runge-Kutta methods and symmetric multistep methods), (cf. [7,9]).

\subsection{Numerical Experiment}

It is of course a natural task to use numerical integrators $\Phi_{h}$ that share several geometric properties with the exact flow of the problem. But does this have any consequences on the global error of the method when it is applied over long time intervals?

The following numerical experiment shows the essential difference between numerical solutions obtained by geometric and non-geometric integrators. We consider the Kepler problem which is Hamiltonian with

$$
H\left(p_{1}, p_{2}, q_{1}, q_{2}\right)=\frac{1}{2}\left(p_{1}^{2}+p_{2}^{2}\right)-\frac{1}{\sqrt{q_{1}^{2}+q_{2}^{2}}},
$$

and we take initial values $q_{1}(0)=1-e, q_{2}(0)=p_{1}(0)=0, p_{2}(0)=$ $\sqrt{(1+e) /(1-e)}$ so that the solution is periodic with period $2 \pi$. This Hamiltonian system has as further invariant the angular momentum

$$
L\left(p_{1}, p_{2}, q_{1}, q_{2}\right)=q_{1} p_{2}-q_{2} p_{1} .
$$

We integrate this problem with $e=0.2$ over a time interval of 100000 periods, and we use a classical explicit method on the one hand and a symplectic and symmetric integrator on the other hand. Both are of order 8 and the (constant) step sizes are such that the numerical work is comparable. For this experiment it is of no importance if we consider composition, Runge-Kutta, or multistep methods. The result is plotted in Fig. 1.

The upper picture shows the global error as a function of time. For the symplectic and/or symmetric method it behaves like $\mathcal{O}\left(t h^{8}\right)$-linear error growth. For a non-symplectic and non-symmetric method of order 8 the global error behaves like $\mathcal{O}\left(t h^{8}+t^{2} h^{9}\right)$ - quadratic error growth for $t>h^{-1}$. For a non-symplectic and non-symmetric method of odd order we would have observed the quadratic error growth already from the beginning of the integration.

The lower picture of Fig. 1 shows the error in the two first integrals of the system. For the symplectic integrator, the angular momentum 

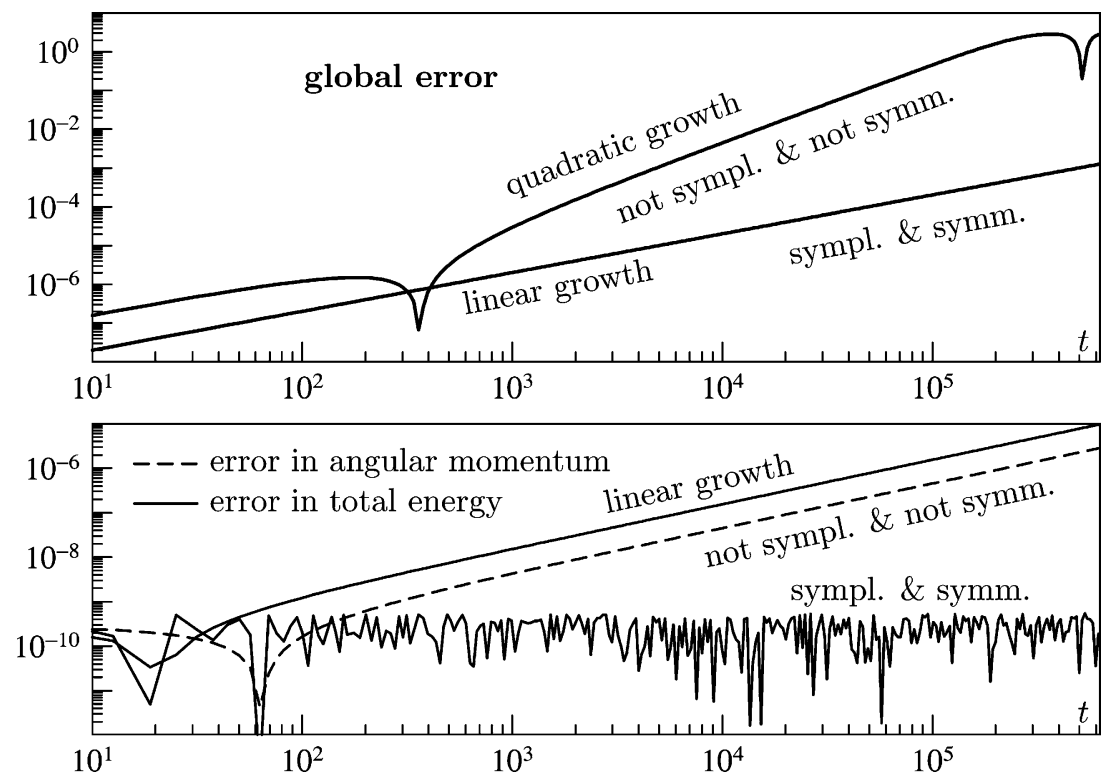

Fig. 1. Long-time behaviour of geometric integrators compared to classical non-symplectic and non-symmetric methods.

is exactly conserved (up to round-off), and the error in the Hamiltonian (total energy) behaves like $\mathcal{O}\left(h^{8}\right)$ and no drift can be observed. For the non-symplectic and non-symmetric method we have a linear drift in the error of the Hamiltonian as well as in that of the angular momentum.

All these statements on the long-time behaviour of geometric integrators can be explained with the help of a backward error analysis. The basic ideas of this theory will be sketched in the following section.

\section{BACKWARD ERROR ANALYSIS}

Backward error analysis is the most important tool for a deeper understanding of the improved long-time behaviour of geometric integrators.

\subsection{Modified Equations}

We start with a general ordinary differential equation $\dot{y}=f(y)$ and an arbitrary numerical method $y_{n+1}=\Phi_{h}\left(y_{n}\right)$. The idea of backward error analysis consists in considering a modified differential equation

$$
\dot{y}=f(y)+h f_{2}(y)+h^{2} f_{3}(y)+\cdots,
$$


such that the exact time- $h$ flow $\widetilde{\varphi}_{h}(y)$ of (15) is formally equal to the numerical flow $\Phi_{h}(y)$. We emphasize that equality has to be understood in the sense of formal power series of $h$, because the series in (15) is in general divergent for all $h>0$.

As an example, consider the pendulum equation

$$
\dot{q}=p, \quad \dot{p}=-\sin q,
$$

which is Hamiltonian with

$$
H(p, q)=\frac{1}{2} p^{2}-\cos q .
$$

The numerical flow of the explicit Euler method $y_{n+1}=y_{n}+h f\left(y_{n}\right)$ is $\Phi_{h}(y)=y+h f(y)$. Developing the exact solution $\widetilde{\varphi}_{h}(y)$ of (15) into powers of $h$ and comparing it to $\Phi_{h}(y)$, yields recurrence relations for the coefficient functions $f_{j}(y)$. For the special case of the pendulum equation this gives

$$
\left(\begin{array}{c}
\dot{q} \\
\dot{p}
\end{array}\right)=\left(\begin{array}{c}
p \\
-\sin q
\end{array}\right)+\frac{h}{2}\left(\begin{array}{c}
\sin q \\
p \cos q
\end{array}\right)+\frac{h^{2}}{12}\left(\begin{array}{c}
-4 p \cos q \\
\left(p^{2}+4 \cos q\right) \sin q
\end{array}\right)+\cdots
$$

Figure 2 (left picture) shows several exact solutions of the truncated modified equation (16) with $h=0.4$. Also included is the numerical solution of the explicit Euler method applied to the initial value that is indicated as a large black dot. We observe that this numerical solution agrees extremely well with the flow of (16).

Next, consider the symplectic Euler (explicit in $q$, implicit in $p$ ) which, for the Hamiltonian system (1), is given by $p_{n+1}=p_{n}-h \nabla_{q} H\left(p_{n+1}, q_{n}\right)$, $q_{n+1}=q_{n}+h \nabla_{p} H\left(p_{n+1}, q_{n}\right)$. The modified equation of this method is

$$
\left(\begin{array}{c}
\dot{q} \\
\dot{p}
\end{array}\right)=\left(\begin{array}{c}
p \\
-\sin q
\end{array}\right)+\frac{h}{2}\left(\begin{array}{c}
-\sin q \\
p \cos q
\end{array}\right)+\frac{h^{2}}{12}\left(\begin{array}{c}
2 p \cos q \\
\left(p^{2}-2 \cos q\right) \sin q
\end{array}\right)+\cdots
$$

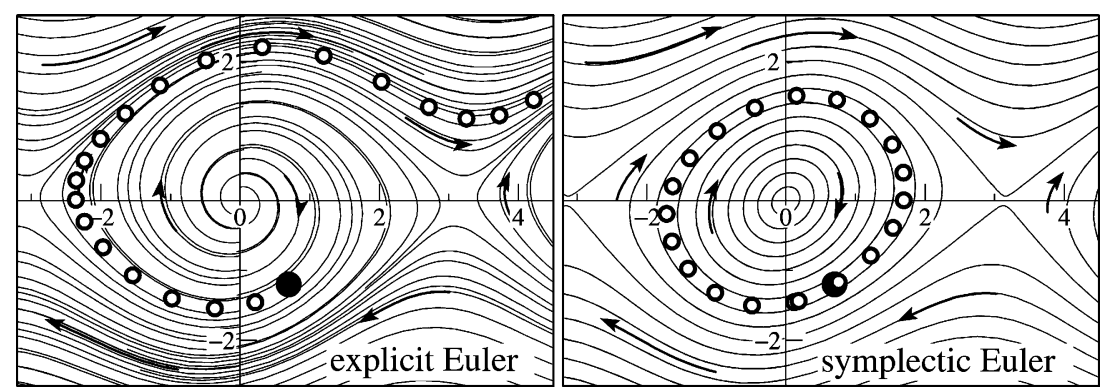

Fig. 2. Numerical solution compared to the exact solution of the truncated modified equation. 
and, similar as before, the right picture of Fig. 2 shows exact solutions of (17) together with a numerical solution of the symplectic Euler method. The important observation is that the system (17) is Hamiltonian with

$$
\widetilde{H}(p, q)=\frac{1}{2} p^{2}-\cos q-\frac{h}{2} p \sin q+\frac{h^{2}}{12}\left(p^{2}-\cos q\right) \cos q+\cdots
$$

This explains why the solution curves are closed. We again observe the excellent agreement of the numerical solution with the flow of (17). Later in this section we shall see that the numerical solution stays close to the level set of (18) for exponentially long times.

\subsection{Hamiltonian Systems}

The observation of the previous numerical experiment is true in general. If one applies any symplectic integrator of order $r$ to a Hamiltonian system (1), then the corresponding modified differential equation is (formally) Hamiltonian with

$$
\widetilde{H}(p, q)=H(p, q)+h^{r} H_{r+1}(p, q)+h^{r+1} H_{r+2}(p, q)+\cdots
$$

The original proof of this result (c.f. $[2,15])$ is based on the integrability lemma, and the existence of the functions $H_{j}(p, q)$ is therefore only of local nature. However, for all symplectic methods of interest (such as the symplectic Euler method, the Störmer-Verlet scheme, and all partitioned Runge-Kutta methods) one can find explicit recurrence relations for the $H_{j}(p, q)$ which show that they are composed of derivatives of $H(p, q)$ and therefore globally defined (mentioned in $[2,13]$, and discussed in detail in Section IX.3.2 of [9]). This has an important consequence for the numerical solution of such symplectic integrators.

Assume for the moment that (15) and (19) are not only formal series but that they are convergent. In this case the flow $\widetilde{\varphi}_{t}(p, q)$ of the modified differential equation would be well defined, and we would have $\left(p_{n}, q_{n}\right)=$ $\widetilde{\varphi}_{h}\left(p_{n-1}, q_{n-1}\right)=\widetilde{\varphi}_{n h}\left(p_{0}, q_{0}\right)$ as well as $\widetilde{H}\left(p_{n}, q_{n}\right)=$ const. This, together with (19), would then imply that

$$
H\left(p_{n}, q_{n}\right)=\text { const }+\mathcal{O}\left(h^{r}\right)
$$

as long as the numerical solution $\left(p_{n}, q_{n}\right)$ stays in a compact set, and the numerical observations of the lower picture of Fig. 1 and of the right picture of Fig. 2 would be completely explained. Unfortunately, the series defining the modified differential equations converges only in exceptional cases and a more subtle analysis is necessary. 


\subsection{Rigorous Estimates of the Local Error}

To make the above analysis rigorous we have to truncate the modified differential equation

$$
\dot{y}=f(y)+h f_{2}(y)+h^{2} f_{3}(y)+\cdots+h^{N-1} f_{N}(y),
$$

so that its flow, denoted by $\widetilde{\varphi}_{N, t}(y)$, becomes well defined. This truncation causes an error in the approximation of the numerical flow and we only have

$$
\left\|\Phi_{h}(y)-\widetilde{\varphi}_{N, h}(y)\right\| \leqslant C_{N}(y) h^{N+1} .
$$

We still have the freedom in choosing the truncation index $N$. In the following we only outline the essential ideas. The details are very technical and can be found in [2,14], and in Chapter IX of [9].

Without any further assumptions on the vector field $f(y)$ and on the coefficient functions of the $h$-expansion of $\Phi_{h}(y)$, it is not possible to get practical estimates for $C_{N}(y)$ in (22). It is convenient to assume these functions to be analytic so that Cauchy's estimates can be used. Choosing $N$ proportional to $(\omega h)^{-1}$ (where $\omega$ is a measure of the Lipschitz constant of $f(y)$ ) makes the bound in (22) minimal and yields

$$
\left\|\Phi_{h}(y)-\widetilde{\varphi}_{N, h}(y)\right\| \leqslant C(y) h \exp \left(-\frac{\gamma}{\omega h}\right)
$$

as long as the step size $h$ is small enough, i.e. $\omega h \leqslant \gamma$. This is the fundamental estimate in rigorous backward error analysis and is the basic ingredient of many results on the long-time behaviour of numerical integrators.

For example, the near-conservation of the Hamiltonian (see the end of Sec. 2.2) can now be proved rigorously. For a symplectic integrator the truncated modified equation is Hamiltonian with

$$
\widetilde{H}_{N}(p, q)=H(p, q)+h^{r} H_{r+1}(p, q)+\cdots+h^{N-1} H_{N}(p, q) .
$$

Since $\widetilde{H}_{N}(p, q)$ is exactly constant along the flow $\varphi_{N, t}(p, q)$, we have by (23) that $\left\|\widetilde{H}_{N}\left(p_{n}, q_{n}\right)-\widetilde{H}_{N}\left(p_{n-1}, q_{n-1}\right)\right\| \leqslant C_{1}\left(p_{n-1}, q_{n-1}\right) h \exp \left(-\frac{\gamma}{\omega h}\right)$ and, summing up, yields $\left\|\widetilde{H}_{N}\left(p_{n}, q_{n}\right)-\widetilde{H}_{N}\left(p_{0}, q_{0}\right)\right\| \leqslant C_{1} n h \exp \left(-\frac{\gamma}{\omega h}\right)$. This then proves (20) on exponentially long time intervals $t=n h \leqslant \exp \left(\frac{\gamma}{2 \omega h}\right)$.

\section{LARGE LIPSCHITZ CONSTANTS}

In the same way as classical convergence results do not yield any insight into the numerical solution of stiff differential equations, the theory of Sec. 2 is useless as soon as $\omega h$ (product of the Lipschitz constant with the step size) is not sufficiently small. We consider the problem 


$$
\ddot{q}+\Omega^{2} q=-\nabla U(q),
$$

where the large Lipschitz constant stems from the linear part in the differential equation. It is Hamiltonian with

$$
H(q, \dot{q})=\frac{1}{2}\|\dot{q}\|^{2}+\frac{1}{2}\|\Omega q\|^{2}+U(q) .
$$

We assume that $\Omega$ is a positive definite symmetric matrix with largest eigenvalue $\omega \gg 1$, and that the derivatives of the potential $U(q)$ are bounded independently of $\omega$. Written as a first order system in the variables $(\Omega q, \dot{q})$, the Lipschitz constant of the resulting system is close to $\omega$.

In the following we consider two situations. First, we treat the case where the eigenvalues of $\Omega$ are all clustered around $\omega$ and 0 . For this case we shortly present the idea of an alternative theory which gives much insight into the long-time behaviour of numerical solutions. Second, we consider space discretizations of nonlinear wave equations (partial differential equations) which lead to systems with a large range of frequencies. We present some numerical experiments with a pseudo-spectral discretization of the sine-Gordon equation.

\subsection{FPU-Type Problems}

As a simplified model for molecular dynamics simulations we consider a chain of alternating stiff harmonic and soft non-linear springs (FermiPasta-Ulam (FPU) type problem, see [8] and Chapter XIII of [9]). This leads to a differential equation of the form:

$$
\begin{aligned}
\ddot{q}_{1} & =-\nabla_{q_{1}} U\left(q_{1}, q_{2}\right), \\
\ddot{q}_{2}+\omega^{2} q_{2} & =-\nabla_{q_{2}} U\left(q_{1}, q_{2}\right),
\end{aligned}
$$

which is of the form (24) where $\Omega$ is diagonal with entries 0 and $\omega \gg 1$. Here, the components of $q_{1}$ denote the displacements from the position of rest of the stiff springs (slow variables), and those of $q_{2}$ denote the expansion/compression of the stiff springs (fast variables).

Besides the Hamiltonian (25) we also consider the oscillatory energy of the individual stiff springs

$$
I_{k}(q, \dot{q})=\frac{1}{2} \dot{q}_{2, k}^{2}+\frac{1}{2} \omega^{2} q_{2, k}^{2}, \quad k=1, \ldots, K .
$$

Here, $q_{2, k}$ is the $k$ th component of $q_{2}$ and $K$ is the number of stiff springs. The interesting fact is (see $[1,3])$ that the sum of the oscillatory energies corresponding to the same large frequency

$$
I(q, \dot{q})=I_{1}(q, \dot{q})+\cdots+I_{K}(q, \dot{q})
$$


is an adiabatic invariant, which means that along solutions of (26) we have $I(q(t), \dot{q}(t))=$ const $+\mathcal{O}\left(\omega^{-1}\right)$ on time intervals of length $\mathcal{O}\left(\mathrm{e}^{\gamma \omega}\right)$. This can be observed in the left picture of Fig. 3, where for $K=3, \omega=100$, initial values satisfying $I_{1}=1, I_{2}=I_{3}=0$, and potential as in [8], the oscillatory energies and the Hamiltonian are plotted along the exact solution.

For the numerical solution of (24) one can in principle apply the Störmer-Verlet method (12). However, the step size is restricted to $\omega h<2$ by stability requirements. A possibility for avoiding such stability restrictions is to consider Gautschi-type methods

$$
q_{n+1}-2 \cos (h \Omega) q_{n}+q_{n-1}=-h^{2} \Psi \nabla U\left(\Phi q_{n}\right) .
$$

Here, $\Psi=\psi(h \Omega), \Phi=\varphi(h \Omega)$ with functions satisfying $\psi(0)=\varphi(0)=1$. Notice that the recurrence (29) produces the exact solution when $U(q)=$ const. Methods of this type have been originally introduced by Gautschi [6] with $\varphi(\xi)=1$ and $\psi(\xi)=\sin c^{2}(\xi / 2)$ (we use the notation $\sin c(\xi)=$ $\sin \xi / \xi)$. Renewed interest on them comes from the article by GarcíaArchilla et al. [5], where such long-time-step methods are considered in view of applications in molecular dynamics simulations. They treat mainly the case where $\varphi(\xi)$ is arbitrary and $\psi(\xi)=\sin c(\xi) \varphi(\xi)$ so that the method is symplectic.

The right picture of Fig. 3 shows the oscillatory and total energies along the numerical solution of (29) with $\psi(\xi)=\sin c(\xi), \varphi(\xi)=1, \omega=100$ and large step size $h=2 / \omega$. The Hamiltonian and the sum (28) are well conserved over long time intervals.

To explain the excellent long-time behaviour of this method, backward error analysis (Sec. 2) is not useful because $\omega h$ is not small. The idea is to write the numerical solution obtained by (29) in the form (for $t=n h$ )

$$
q_{n}=y_{h}(t)+\sum_{k \neq 0} \mathrm{e}^{\mathrm{i} k \omega t} z_{h}^{k}(t)
$$
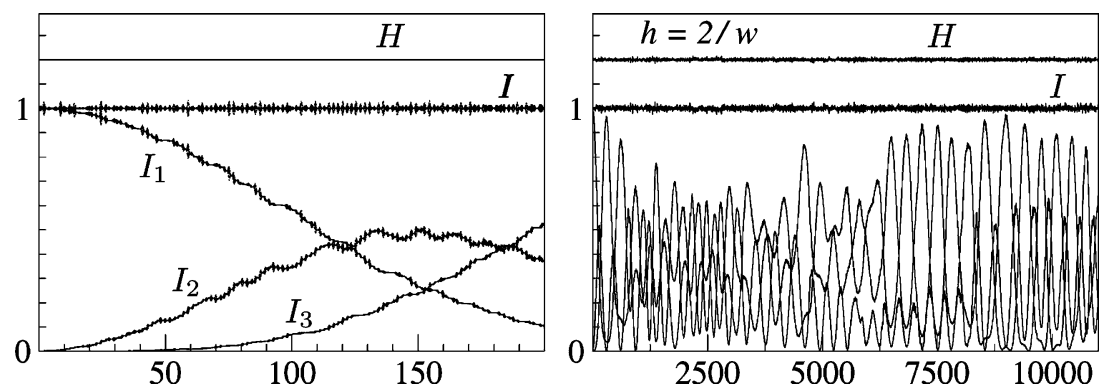

Fig. 3. Oscillatory and total energies along the exact solution (left) and along the numerical solution obtained by a Gautschi-type method with large step size $h=2 / \omega$ (right). 
with smooth coefficient functions. Such an expansion has been proposed in [8] and is called modulated Fourier expansion in [9]. Inserting (30) into the method (29) and comparing the coefficients of $\mathrm{e}^{\mathrm{i} k \omega t}$ yields differential equations for $y_{h}(t)$ and $z_{h}^{k}(t)$ which are of singular perturbation type. The coefficient functions of (30) are then the smooth (i.e. non-oscillating) solutions of this system. A detailed study (see Chapter XIII of [9]) shows that the $z_{h}^{k}(t)$ decay like $\omega^{-|k|}$ as $|k| \rightarrow \infty$, and that the differential equation for these functions has two formal first integrals (corresponding to $H$ and to $I$ ). This allows one to prove the following result.

Under suitable assumptions on the differential equation (analyticity of $U(q)$, initial values satisfying $\frac{1}{2}\left\|\dot{q}_{0}\right\|^{2}+\frac{1}{2}\left\|\Omega q_{0}\right\|^{2} \leqslant E$ with $E$ independent of $\omega$ ), on the method (conditions on $\psi(\xi)$ and $\varphi(\xi)$ ), and on the step size $\left(h \omega \geqslant c_{0}>0, h \leqslant h_{0}\right.$, and the non-resonance condition $\left|\sin \left(\frac{1}{2} h k \omega\right)\right| \geqslant c \sqrt{h}$ for $k=1, \ldots, N$ ) one can prove that

$$
\begin{aligned}
H\left(q_{n}, \dot{q}_{n}\right) & =H\left(q_{0}, \dot{q}_{0}\right)+\mathcal{O}(h), \\
I\left(q_{n}, \dot{q}_{n}\right) & =I\left(q_{0}, \dot{q}_{0}\right)+\mathcal{O}(h)
\end{aligned}
$$

for $0 \leqslant n h \leqslant h^{-N+1}$. We mention that these techniques do not allow to prove the near energy conservation without considering at the same time also that of the oscillatory energy.

The numerical non-resonance condition $\left|\sin \left(\frac{1}{2} h k \omega\right)\right| \geqslant c \sqrt{h}$ (for $k=$ $1, \ldots, N)$ excludes that $h \omega$ is $o(\sqrt{h})$ close to integral multiples of $\pi$, and defines via the integer $N$ the length of interval where (31) holds. Without this technical assumption the analysis is much more complicated, and the conservation of the total and/or oscillatory energies strongly depends on the choice of the filter functions $\varphi(\xi)$ and $\psi(\xi)$ (see [8] for a detailed discussion in the case of a quadratic potential).

Similar results can be obtained for the situation where the eigenvalues of $\Omega$ are $\left\{0, a_{1} \omega, \ldots, a_{s} \omega\right\}$ with fixed $a_{j}$ and $\omega \gg 1$ (see [4]). If the $a_{j}$ are rationally independent, the oscillatory energies of the individual frequencies are well conserved. In the presence of resonances among the $a_{j}$, an energy exchange can take place on a scale depending on the kind of resonance.

\subsection{Sine-Gordon Equation}

A situation, neither covered by the theory of Sec. 2 nor by that of Sec. 3.1, is the space discretization of non-linear wave equations. Following the experiment of Hochbruck and Lubich [12] we consider the sine-Gordon equation:

$$
u_{t t}=u_{x x}-\sin u
$$


for $-1 \leqslant x \leqslant 1$ and $t \geqslant 0$ subject to periodic boundary conditions. Pseudospectral discretization in space with equidistant collocation points $x_{j}=$ $2 j / N(j=-N / 2, \ldots, N / 2)$ yields an approximation

$$
\widehat{u}(x, t)=\sum_{|k| \leqslant N / 2}^{\prime} q_{k}(t) \mathrm{e}^{\mathrm{i} k \pi x}
$$

(the prime on the sum indicates that the first and last summands are multiplied by $1 / 2)$, where the $N$-periodic sequence $q(t)=\left(q_{k}(t)\right)$ satisfies

$$
\ddot{q}+\Omega^{2} q=-\mathcal{F}_{N} \sin \left(\mathcal{F}_{N}^{-1} q\right),
$$

$\mathcal{F}_{N}$ denotes the discrete Fourier transform, and $\Omega$ is a diagonal matrix with entries $\omega_{k}=k \pi$ for $|k| \leqslant N / 2$. Introducing the velocity $p=\dot{q}$, the system (33) is seen to be Hamiltonian with

$$
H(p, q)=\frac{1}{2} p^{*} p+\frac{1}{2} q^{*} \Omega^{2} q+V(q), \quad V(q)=N \sum_{|k| \leqslant N / 2}^{\prime}\left(1-\cos U_{j}\right),
$$

where $U=\left(U_{j}\right)=\mathcal{F}_{N}^{-1} q$. We are interested in numerical methods that nearly conserve this Hamiltonian over long time intervals.

Motivated by the analysis of Sec. 3.1 we also consider the oscillatory energies

$$
I_{k}(p, q)=\frac{1}{2}\left|p_{k}\right|^{2}+\frac{1}{2} \omega_{k}^{2}\left|q_{k}\right|^{2}
$$

of the individual frequency modes. With initial functions

$$
\begin{aligned}
u(x, 0) & =\pi, \\
u_{t}(x, 0) & =\sin (\pi x)+0.005 \pi^{2}\left(1-x^{2}\right)
\end{aligned}
$$

the Hamiltonian $H(p, q)$ and the oscillatory energies $I_{k}(p, q)$ are plotted in Fig. 4 along the exact solution of (33) with $N=128$. The thick line (with constant value $\approx 2.5$ ) is the Hamiltonian, $I_{0}$ is the curve oscillating between 1 and $10^{-3}, I_{1}$ is nearly constant with a value close to $0.5, I_{2}$ is essentially oscillating between $10^{-4}$ and $10^{-5}$, and the further oscillatory energies appear in decreasing order. It came as a surprise to us that most of the oscillatory energies are very close to being constant similar as what has been observed for (28) in the FPU-type problem.

We apply the explicit, variable step size Runge-Kutta code DOPRI5 (see [11]) with tolerance $T o l=2 \times 10^{-4}$ to the differential equation (33) on the interval $[0,550]$. This integration takes 103953 accepted steps. The Hamiltonian and the oscillatory energies along the numerical solution, plotted in Fig. 5, are not correct. In particular, the oscillatory energy corresponding to the high-frequency modes do not remain small, but increase 


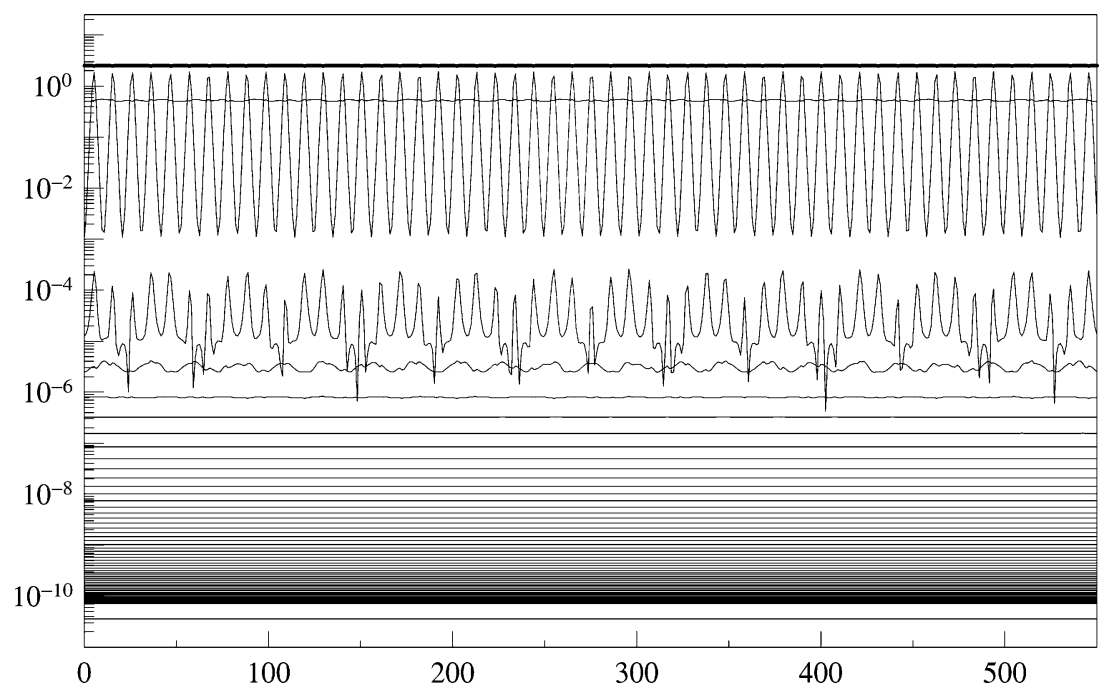

Fig. 4. Hamiltonian (34) and oscillatory energies (35) along the exact solution of the differential equation (33).

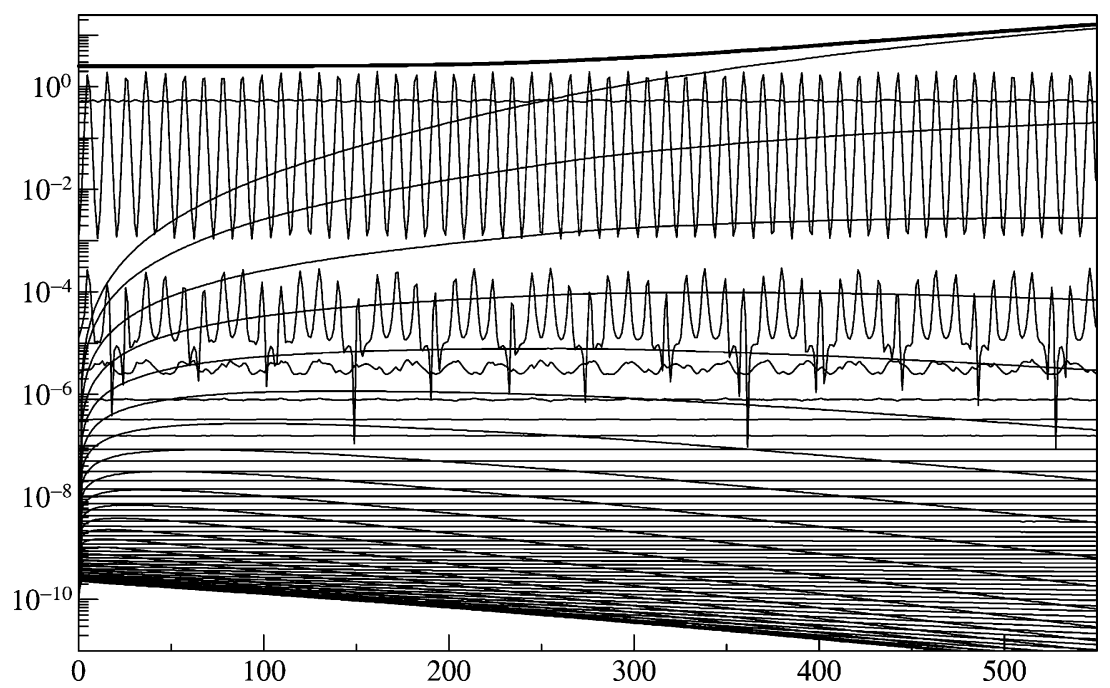

Fig. 5. Hamiltonian (34) and oscillatory energies (35) along the numerical solution of (33) obtained with DOPRI5. 

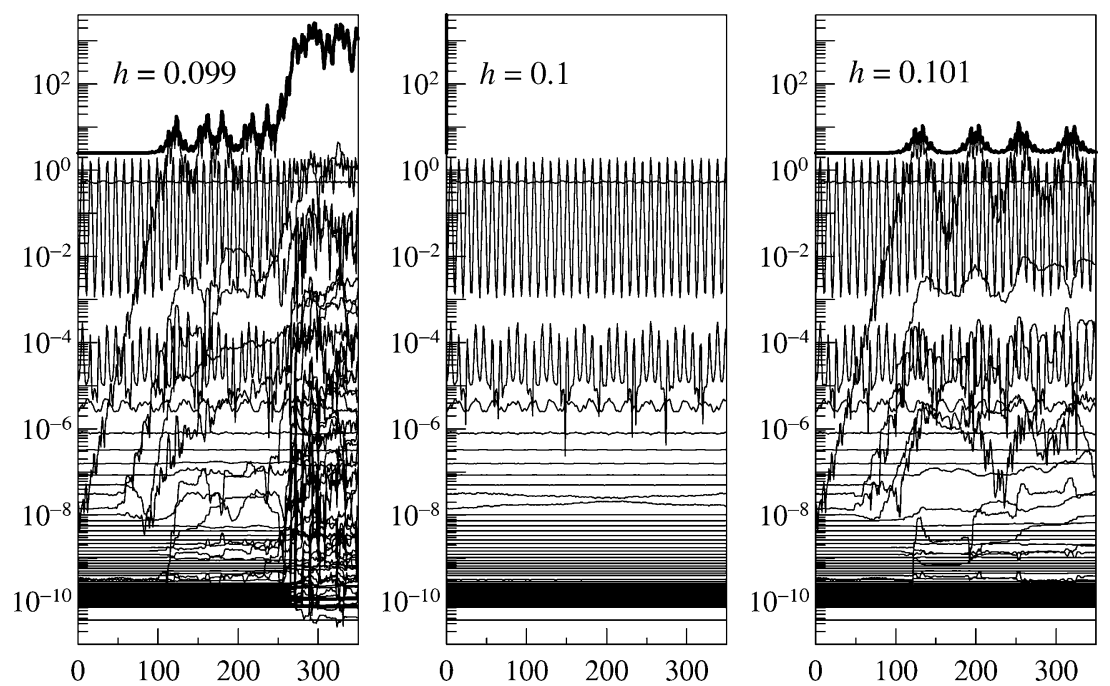

Fig. 6. Hamiltonian (34) and oscillatory energies (35) along the numerical of (33) obtained with method $\left(29, \varphi(\xi)=1\right.$ and $\psi(\xi)=\sin c^{2}(\xi / 2)$, and with three different step sizes.

rapidly until they reach a value close to Tol. Some of them continue to increase slowly and give rise to an unacceptable error in the Hamiltonian. The oscillatory energy for the low frequency modes are well reproduced in this experiment.

We finally apply the Gautschi-type method of Sec. 3.1 with several different choices of the filter functions to the differential equation (33), again with $N=128$. We use the constant step size $h=0.1$ so that only 5500 steps yield an approximation on the same interval as before. In our experiments we observe that whenever the function $\psi(\xi)$ contains $\sin c(\xi)$ as factor, i.e. it vanishes at all integral multiples of $\pi$, the simulation gives a result that cannot be distinguished from that of the exact solution in Fig. 4. Since the frequencies of the discretized sine-Gordon equation are in resonance and without any gaps, this is an unexpected long-time behaviour.

Figure 6 shows the same experiment for the original method of Gautschi $\left(\varphi(\xi)=1\right.$ and $\left.\psi(\xi)=\sin c^{2}(\xi / 2)\right)$, for which the filter function $\psi(\xi)$ does not vanish at odd integral multiples of $\pi$. In this case, the energies are wrongly reproduced, and they are very sensitive with respect to small changes in the step size. This does not seem to be the case when $\psi(\xi)$ contains the factor $\sin c(\xi)$. It would be of interest to get more insight into the long-time behaviour of these methods. 


\section{ACKNOWLEDGMENTS}

This paper was presented at the AFOSR workshop "Advances and Challenges in Time-Integration of PDE's", held in August 18-20, 2003, at Brown University, RI, USA. The author is grateful to the organizers for providing the stimulating atmosphere at this workshop.

\section{REFERENCES}

1. Benettin, G., Galgani, L., and Giorgilli, A. (1987). Realization of holonomic constraints and freezing of high frequency degrees of freedom in the light of classical perturbation theory. Part I. Comm. Math. Phys. 113, 87-103.

2. Benettin, G., and Giorgilli, A. (1994). On the Hamiltonian interpolation of near to the identity symplectic mappings with application to symplectic integration algorithms. J. Statist. Phys. 74, 1117-1143.

3. Cohen, D., Hairer, E., and Lubich, C. (2003). Modulated Fourier expansions of highly oscillatory differential equations. Found. Comput. Math. 3, 327-345.

4. Cohen, D., Hairer, E., and Lubich, C. (2004). Numerical energy conservation for multifrequency oscillatory differential equations. To appear in BIT.

5. García-Archilla, B., Sanz-Serna, J. M., and Skeel, R. D. (1999). Long-time-step methods for oscillatory differential equations. SIAM J. Sci. Comput. 20, 930-963.

6. Gautschi, W. (1961). Numerical integration of ordinary differential equations based on trigonometric polynomials. Numer. Math. 3, 381-397.

7. Hairer, E., and Hairer, M. (2003). GniCodes - Matlab programs for geometric numerical integration. In: Frontiers in Numerical Analysis (Durham 2002), Springer, Berlin.

8. Hairer, E., and Lubich, C. (2000). Long-time energy conservation of numerical methods for oscillatory differential equations. SIAM J. Numer. Anal. 38, 414441.

9. Hairer, E., Lubich, C., and Wanner, G. (2002). Geometric Numerical Integration. Structure-Preserving Algorithms for Ordinary Differential Equations. Springer Series in Computational Mathematics 31. Springer, Berlin.

10. Hairer, E., Lubich, C., and Wanner, G. (2003). Geometric numerical integration illustrated by the Störmer-Verlet method. Acta Numerica 12, 399-450.

11. Hairer, E., Nørsett, S. P., and Wanner, G. (1993). Solving Ordinary Differential Equations I. Nonstiff Problems. Springer Series in Computational Mathematics 8, 2nd ed., Springer, Berlin.

12. Hochbruck, M., and Lubich, C. (1999). A Gautschi-type method for oscillatory secondorder differential equations. Numer. Math. 83, 403-426.

13. Murua, A. (1994). Métodos simplécticos desarrollables en P-series. PhD thesis, Univ. Valladolid.

14. Reich, S. (1999). Backward error analysis for numerical integrators. SIAM J. Numer. Anal. 36, 1549-1570.

15. Tang, Y.-F. (1994). Formal energy of a symplectic scheme for Hamiltonian systems and its applications I. Comput. Math. Applic. 27, 31-39. 